\title{
25. JOSÉ VÁZQUEZ ESTÉVEZ (1867-1926) CORRESPONSAL DE BALTASAR MERINO (1845-1917).
}

Jesús IZCO

En el prólogo de su Flora descriptiva é ilustrada de Galicia, B. Merino reconoce el auxilio que varios amigos le han prestado «preparando, enviándonos ó poniendo á nuestra disposición sus colecciones de plantas» ${ }^{1}$. Cita a Marcelino Rodríguez Franco, Primo Castro Pita, Manuel Lago y González, José Casal y Lois, Ricardo Sánchez Varela y a la familia de Victor López Seoane, que cedió materiales recogidos por éste. Si estos fueron los primeros y más generosos, sin duda no fueron los únicos colaboradores. Para X.A. Fraga «un elemento substantivo era a colaboración de antigos alumnos, cregos das parroquias rurais e persoas diversas, que lle facilitaban informacións, acollian, guiaban e fornecian de exemplares» ${ }^{2}$, entre ellos, J. Gómez Martínez, hace figurar a José Vázquez Estévez, «al que hubo de dedicar el autor de la «Flora de Galicia» una [conocemos, al menos, dos dedicatorias] de las especies remitidas ${ }^{3}$.

En efecto, a partir de las adiciones a la Flora, que fueron publicadas en Broteria ${ }^{4}$, son relativamente frecuentes las referencias a la colaboración de José Vázquez Estévez como origen de distintas muestras, algunas de las cuales dieron lugar a la descripción de novedades por parte de B. Merino, quien agradece la colaboración con la dedicatoria de una variedad de Lepidium heterophyllum Bth., y de la especie de Artemisia L. que comentaré luego. En total se contabilizan poco más de 30 taxones en los que B. Merino menciona material suministrado por J. Vázquez; indefectiblemente procedentes de Arbo (Pontevedra), un listado de éstos se incluye en el anexo.

José Vázquez Estévez (Arbo, Pontevedra,
1867-1926) se licenció en Derecho por la Universidad de Santiago, obtuvo el título de doctor el 28 de junio de 1905, con un trabajo titulado La soberanía política. Su concepto, origen, transmisión y legitimidad de su ejercicio, publicado posteriormente ${ }^{5}$, fue profesor de Derecho Canónico; aparte de su ejercicio profesional, participó de forma intensa en la organización agraria desde su condición de propietario y llegó a fundar diversos sindicatos agrarios en la comarca de Tui; añade A. Couceiro Freijomil ${ }^{6}$ que dejó inédito un poema de 164 versos, titulado La vida del campo. Un nieto del corresponsal, Gonzalo Vázquez Uña, conserva, en su archivo particular, algunas de las cartas cruzadas entre B. Merino y J. Vázquez, se trata de cuatro documentos que corresponden a un período comprendido entre julio de 1915 y octubre de 1916, contienen información y observaciones sobre materiales recolectados por el corresponsal además de temas propios de la comunicación escrita entre amigos personales; no se conoce de J. Vázquez ninguna actividad botánica propia independiente, aunque según su nieto (com. pers.) dejó su herbario a la Universidad de Santiago, tal vez copia del que envió a Merino: «Es digno de alabanza y admiración el decidido e infatigable empeño con que nuestro buen amigo se ha propuesto recoger cuantas plantas fanerogámicas vivan cerca de Arbo y reunirlas en colección. Este año, en pocos meses ha juntado más de 600 especies de muestras duplicadas, formando dos hermosas colecciones, una de las cuales ha puesto a nuestra disposición»?

El documento más antiguo de los mencionados tiene fecha de 14 de julio de 1915 
y no lleva membrete ni localidad que lo identifique, la letra corresponde incuestionablemente a J. Vázquez; en este documento, bajo el epígrafe "Artemisia», se describen las diferencias entre distintas plantas que empiezan a florecer en esa fecha; aunque se contrapone una planta al resto, parece que toda ellas se consideran pertenecientes a la misma especie. La diagnosis es la siguiente:

«1 ${ }^{\text {a }}$. Se notan algunas diferencias en las lacinias de las hojas.

$2^{a}$. La flor de una planta aparece notoriamente más blanca que la de las otras dos y las ramillas florales más cortas.

$3^{a}$. Los glomérulos de una planta salen de la axila de una hojita que es más corta y más estrecha y más pelosa q. la de las otras dos plantas; además dicha hoja es aquillada por el dorso hasta el ápice, y las otras dos plantas no, presentándose muy acanalada la de estas en toda su extensión y con el ápice rojizo visto a la lupa.

$4^{\text {a }}$ Presentan muchas más hojitas en los glomérulos las otras dos plantas.» ${ }^{8}$

El material le ha interesado a B. Merino. Desde el Colegio Apóstol Santiago se dirige a J. Vázquez solicitando nuevas muestras de la planta: «Y va de encargo. Mucho le agradecería una rama o tallo de la Artemisia vulgaris y si fuese posible la panoja floral. Llega el tiempo de hacer la descripción de la otra Artemisia.» ${ }^{9}$

Meses más tarde vuelve J. Vázquez sobre el mismo tema, esta vez con datación en Arbo:

«Supongo que V. habrá observado que los estolones tiernos de la A. sp tienen una especie de escamas; escamas que permanecen algun tiempo aun despues de brotar el estolón y de tener las plantitas 8 o más hojas. Este caracter lo he observado en todos los estolones que hoy he examinado. Me apresuro a participarle esto por si V. lo quiere aprovechar en la descripción de la planta.

Y basta ya de A. sp, hasta que los aquenios esten en sazón.» ${ }^{10}$

$\mathrm{Y}$ en un post scriptum «Acompaño muestrita de los estolones de la Art. sp con las escamas.»

Estos son los antecedentes de la Artemisia vazqueziana Merino, encontrada por J. Vázquez y dedicada por el jesuita «a él tan benemérito de la Flora gallega gustosísimos la dedicamos» ${ }^{11}$. Hay que notar que la descripción original recoge la presencia de un estolón escamoso, carácter que figura en las anotaciones de J. Vázquez. Para Bellot, el material de Arbo "indudablemente, se trata de una especie exótica: la Artemisia verlotorum (sic) Lamot, pues los tres caracteres distintivos de la Artemisia vazqueziana: hojas más angostas, planta estolonífera y cabezuelas rojizas, son los que separan a la Artemisia verlotorum de Lamot. Según nos comunica verbalmvente "L.P. Laínz, Pau ya habia señalado este error del P. Merino" 12.

En las cartas hay mención de otras plantas, J. Vázquez apunta otras observaciones minuciosas, bien sea de un Eupatorium cannabinum L. «que por la forma de sus hojas se diferencia mucho del tipo», o de variaciones en Saponaria L. «unas con las anteras negras, otras con ellas vinosas y otras con ellas blancas». Reclama asimismo información sobre envíos anteriores: « $i Y$ aquella muestra de cicuta que le entregué en Camposancos ¿no mereció ser tenida en cuenta?», de la que tiene ejemplares de cerca de tres metros: «PParece una caña de pescar!» ${ }^{13}$.

B. Merino es igualmente explícito en su correspondencia: «Acabo de recibir su grata con la planta que a $\mathrm{V}$. ha mareado por lo que dice. Es indudablemente el Erysimum linifolium J. Gay. Los sépalos por más que esten erguidos y juntos no estan solapados entre sí, siendo por consiguiente polisépalo el 
cáliz» ${ }^{14}$. Sin duda hay respuesta inmediata de J. Vázquez, aunque falte el documento material. Diez días más tarde B. Merino insiste: «Y vamos a la cruciácea o crucifera de flores violáceas. Si V. viendo toda la planta y comparándola con el Erysimum linifolium la encuentra diferente vaya anotando las diferencias y aunque habra que esperar a que tenga el fruto bien formado y maduro, donde aparecen los caracteres mas distintivos de las cruciáceas, bueno es conservar algunas flores. Pudiera ser alguna Matthiola.» En esta misma carta, B. Merino comunica a J. Vázquez sus proyectos respecto a materiales suministrados previsiblemente por éste: «La Lavandula con los verticilos inferiores hacia el medio del pedúnculo también pienso indicarla en las Adiciones como forma manicata; no existiendo ahí, a lo que creo, la $\underline{L}$. stoechas L. me inclino ahora que sea una forma de la especie típica. La cicuta, si bien no abunda en Galicia, la he visto en varios parajes.» ${ }^{15}$

Del conjunto de la correspondencia se aprecia una estrecha amistad que va mucho más allá de la contraída por razones estrictamente botánicas. Nada extraño si tenemos en cuenta el lugar de nacimiento y de residencia de J. Vázquez, así como de parte de su actividad en el territorio tudense que incluye La Guardia, donde se asentaba el Colegio Santiago Apóstol. Existen evidencias de ello a partir de comentarios de trasfondo político y despedidas con recuerdos a familiares, valgan algunos ejemplos como testimonio:

«Hoy esperamos que llegue la primera comisión de examinadores, que no sé como calificarla, pues todo cuanto hacen y han hecho los demás años es abiertamente contra la Constitución, la cual no exige más prueba oficial que la que precede a la obtención del título profesional: pero evidentemente cuanto más liberales más tiránicos. Llegará un día, así lo creo, en que podamos sacudir tan pesado yugo... Recuerdos a su familia, al Sr.
Comesaña...» ${ }^{16}$

«Recuerdos a los de su casa y a D. Vicente ... Gonzalito va triunfando en toda la línea; hasta ahora lleva 3 sobresalientes en Algebra, Preceptiva y Dibujo. Que sea enhorabuena» ${ }^{17}$

\section{Anexo. J. Vázquez colector de B. Merino.}

El listado recoge las plantas, citadas en la obra de B. Merino, en la que J. Vázquez figura como recolector; todas proceden de Arbo (Pontevedra).

Agrimonia odorata Mill., Broteria 11: 184.

Amaranthus paniculatus L., Broteria 14: 160. Armeria rigida Wallr., Broteria 12: 108.

Artemisia vazqueziana Merino, Broteria 14: 170.

Bryanthus (Daboecia) polifolius Don, f. abnormis Merino, Broteria 12: 166.

Echium plantagineum L. subvar. micranthum Rouy, Broteria 12: 166.

Echium rosulatum L. var. latifolium Merino, Broteria 12: 47.

Erica arborea L. lusus glandulifera, Broteria 14: 166.

Galactites tomentosa Moench. var. candida Merino, Broteria 14: 29.

Galium teres Merino, Broteria 12: 113.

Heracleum sphondilium L. var. elatius Rouy, Broteria 11: 54.

Hieracium boreale Fries diversas var., Broteria 13: 46.

Hieracium rigidum Hartm. f. amygdalinum Rouy, Broteria 13: 45.

Jacobea hirta L., Broteria 12: 170.

Laserpitium prutenicum L. var. dufourianum Rouy et Cam., Broteria 11: 188-189.

Laserpitium prutenicum L. var. parviflorum Car. et Saint-Lag., Broteria 11: 188.

Latyrus angulatus L., Broteria 11: 201.

Lavandula pedunculata Cav. f. candida Merino, Broteria 12: 98.

Lepidium heterophyllum Bth. var. vazquezii Merino, Broteria 11: 110.

Leucanthemum vulgare Lamk. raza sylvatica Hoffg. et Link,, Broteria 12: 176.

Mentha aquatica L. var. glabrata Benthm., Broteria 12: 51 . 
Plantago bellardi All., Broteria 12: 110.

Plantago lagopus L. var. lusitanica L., Broteria 12:

110.

Polygonum orientale L., Broteria 14: 159.

Potentilla tomentilla Sibth. f. polypetala Merino, Broteria 11: 184.

Ranunculus steveni Andraz., Broteria 11: 57.

Scabiosa columbaria L. diversas var., Broteria 13: 50.

Solidago virga-aurea L., Broteria 12: 168.

Sonchus tenerrimus L. var. annus Lange, Broteria 13: 36 .

Stenactis annua Nees, Broteria 12: 168.

Taraxacum officinale Wiggers. var. laevigatum DC., Broteria 13: 34.

Tolpis umbellata Bert. f. latifolia Merino, Broteria 13: 30 .

Veronica anagallis L. var. anagallidiformis Franch, Broteria 12: 45.

Vincetoxicum officinale Moench. var. puberılum Gaunt., Broteria 12: 34.

\section{Notas.}

1. B. Merino. Flora descriptiva é ilustrada de Galicia. Santiago, 1905-1909. 3 vols. El texto citado en vol. 1: VI.

2. Artículo biográfico de B. Merino. En: X.A. Fraga Vázquez \& A. Domínguez (coords.) Diccionario histórico das Ciencias e das Técnicas de Galicia. Autores, 1868-1936: 220-225. A Coruña, 1993. El entrecomillado en pág. 222.

3. J. Gómez Martínez. Arq. Seminar. Estud. Galegos, seizon Ci., 5: 47-63. 1930.

4. B. Merino. Broteria, 10: 125-140; 10: 173-191; I1: 33-59; 11: 105-120; 11: 182-201; 12:32-52; 12: $97-114 ; 12: 163-176 ; 13: 17-32 ; 14: 25-28$; 14: 158-171. 1911-1916.

5. J. Vázquez Estévez. La soberanía política. Su concepto, origen, transmisión y legitimidad de su ejercicio. Túy, 1906.

6. A. Couceiro Freijomil. Diccionario biobibliográfico de escritores. Santiago, 1954; quien añade: «colaboró con el P. Merino, quien lo cita con elogio en la Flora de Galicia, por haber descubierto ciertas variedades de plantas, algunas de las cuales le dedicó el ilustre jesuita» (cf. vol. 3: 470).

7. B. Merino. Broteria 11:182-201 (la cita en pp. 184-185).

8. [J. Vázquez] a B. Merino. [s.1.], 14-VII-1915. Archivo Gonzalo Vázquez Uña (= Arch. G.V.U.).

9. B. Merino a J. Vázquez. La Guardia, 11-VI-1916 (Arch. G.V.U.).

10. J. Vázquez a B. Merino. Arbo, 15-X-1916 (Arch. G.V.U.).

11. B. Merino. Broteria, 14: 170. 1916.

12. F. Bellot \& B. Casaseca. Trab. Jard. Bot. Santiago, 7: 19-22. 1954.

13. J. Vázquez a B. Merino. Arbo, 15-X-1916 (Arch. G.V.U.).

14. B. Merino a J. Vázquez. La Guardia, 1-VI-1916 (Arch. G.V.U.).

15. B. Merino a J. Vázquez. La Guardia, 11-VI1916 (Arch. G.V.U.).

16. B. Merino a J. Vázquez. La Guardia, 1-VI-1916 (Arch. G.V.U.).

17. B. Merino a J. Vázquez. La Guardia, 11-VI1916 (Arch. G.V.U.).

Agradecimientos. A Gonzalo Vázquez Uña por la correspondencia aportada; a Carolina Rovira, por su colaboración para definir profesionalmente al colaborador de Merino; a Antonio González Bueno por revisar y pulir el original.

Aceptado para su publicación en Febrero de 1996

Dirección del autor. Departamento de Biología Vegetal. Facultad de Farmacia. E-15706 Santiago de Compostela. 